\title{
A Case of posterior reversible encephalopathy syndrome in a pregnant woman with sickle cell anemia
}

\author{
Ravi Zala ${ }^{1, *}$, Bhavesh Airoa ${ }^{2}$, Panshit Savalia ${ }^{3}$, Manish R. Pandya ${ }^{4}$ \\ ${ }^{1,3}$ Resident, ${ }^{2}$ Associate Doctor, ${ }^{4}$ Professor \& HOD, ${ }^{1,2,3}$ Dept. of Obstetrics and Gynecology, C. U. Shah Medical College and \\ Hospital, Gujarat, ${ }^{4}$ Scientific Research Institute
}

*Corresponding Author:

Email: drravizala2503@gmail.com

\begin{abstract}
Posterior reversible encephalopathy syndrome (PRES) is a clinical entity characterized by headache, altered mental functioning, seizures and loss of vision associated with white matter changes predominantly present in the posterior cerebral lobe. ${ }^{1}$

Hereby I am reporting a 23 year old primigravida with 8 months of amenorrhoea who was a K/C/O Sickle cell disease who developed eclampsia, underwent Caesarean for the same and developed PRES syndrome post op. She was treated with antihypertensives and antiepileptics and gradually improved over time. Early diagnosis and prompt intervention stays the mainstay of this syndrome, because once the irreversible changes set it, complete resolution to normal is not possible.
\end{abstract}

Keywords: Posterior reversible encephalopathy syndrome, Reversible posterior leukoencephalopathy syndrome, Postpartum, Sickle cell disease, Pregnancy.

\section{Introduction}

Although most pregnancies complicated by maternal sickle cell disease (SCD) are likely to result in live birth, these pregnancies are at increased risk of obstetrical and fetal complications, as well as medical complications of SCD. These risks are due, at least in part, to the metabolic demands, hypercoagulable state, and vascular stasis associated with pregnancy. Patients suffering from SCD are more prone to develop preeclampsia and eclampsia due to sickle cell nephropathy.

Posterior reversible encephalopathy syndrome (PRES) also known as Reversible posterior leukoencephalopathy syndrome (RPLS) first came into picture in 1996 when Dr. Judy Hinchey and her colleagues through their studies from the year 1988 to 1994 at New England Medical Center in Boston and Hospital Sainte Anne in Paris noted a characteristic syndrome comprising of headache, altered mental functioning, seizures and loss of vision associated with white matter changes predominantly present in the posterior cerebral lobe. ${ }^{[1]}$ Despite its name this syndrome is not always reversible. PRES being such a rare condition, is often missed while considering the differential diagnosis causing delay in treatment which can lead to permanent neurological damage.

\section{Case Report}

A 23 year old primigravida, a K/C/O Sickle Cell Disease with 8 months of amenorrhea presented to the Outpatient Department of Obstetrics and Gynecology with complaints of generalized body ache and weakness associated with bilateral pedal edema since 3 to 4 days. On examination, she was conscious, cooperative and well oriented to time, place and person. Her blood pressure at time of presentation was $160 / 110 \mathrm{mmHg}$.
On general examination, pallor and bilateral pedal edema was found to be present. Respiratory system examination revealed crepitations in left lung field. CNS \& CVS examination revealed no abnormal findings. At the time of presentation her Hemoglobin level, total count and platelet count were $6.1 \mathrm{~g} / \mathrm{dl}$, $17,600 / \mathrm{mm}^{3}$ and $3,04,0001 \mathrm{ac} / \mathrm{mm}^{3}$ respectively. Urine examination revealed +1 proteinuria

Patient was admitted in Medicine ward with the suspicion of ongoing sickle cell crisis. Adequate hydration was maintained along with $\mathrm{O}_{2}$ support and injectable antibiotics in the view of preventing further sickling. Anemia was corrected by administering 2 units of packed cell volume and patient was put on Tab labetalol TDS. Despite all the antihypertensive treatment she had 2 episodes of eclamptic convulsions on day 3 of admission. Decision of terminating the pregnancy was made. Hence induction of labor was done with tablet misoprostol $50 \mathrm{mcg}$ PV stat after giving $4 \mathrm{gm} \mathrm{MgSO}_{4} \mathrm{I} . \mathrm{V}$. and 3 gram in each buttock followed by 2 maintenance doses 5 gram each 6 hours apart. She had $3^{\text {rd }}$ convulsion 5 hours after induction of labor hence decision for Caesarean Section was taken. LSCS was uneventful. Patient had $4^{\text {th }}$ convulsion 4 hours after C.Section which was characterised by twitching of all four limbs, uprolling of eyeballs, and frothing from mouth. Inj mannitol and Inj Hydrocortisone was given. On physician advice, Inj Valproate was given once. In Spite of all these measures $4^{\text {th }}$ convulsive episode occurred and then patient was put on Tab Valproate $500 \mathrm{mg}$ BD. Once vitally stable, she underwent MRI which depicted hyperintense signals on T2 weighted and FLAIR(Fluid Attenuated Inversion Recovery) images and hypo intense on $\mathrm{T} 1$ weighted (images involving cortical and subcortical frontal posterior parietooccipital lobes and 
basal ganglia. Diffusion Weighted Images didn't reveal any diffusion restriction in these areas. A diagnosis of PRES was made and was managed by maintaining blood pressure <140/90 mmHg using Tablet Nifedipine. Tablet Valproate was used prophylactically in BD dose to avoid any further convulsions. Adequate hydration was maintained and proper antibiotic coverage was given to avoid sickling. Patient recovered completely and was discharged on postop day 9. On follow up MRI, there was complete resolution of previously noted abnormal signals suggestive of PRES.
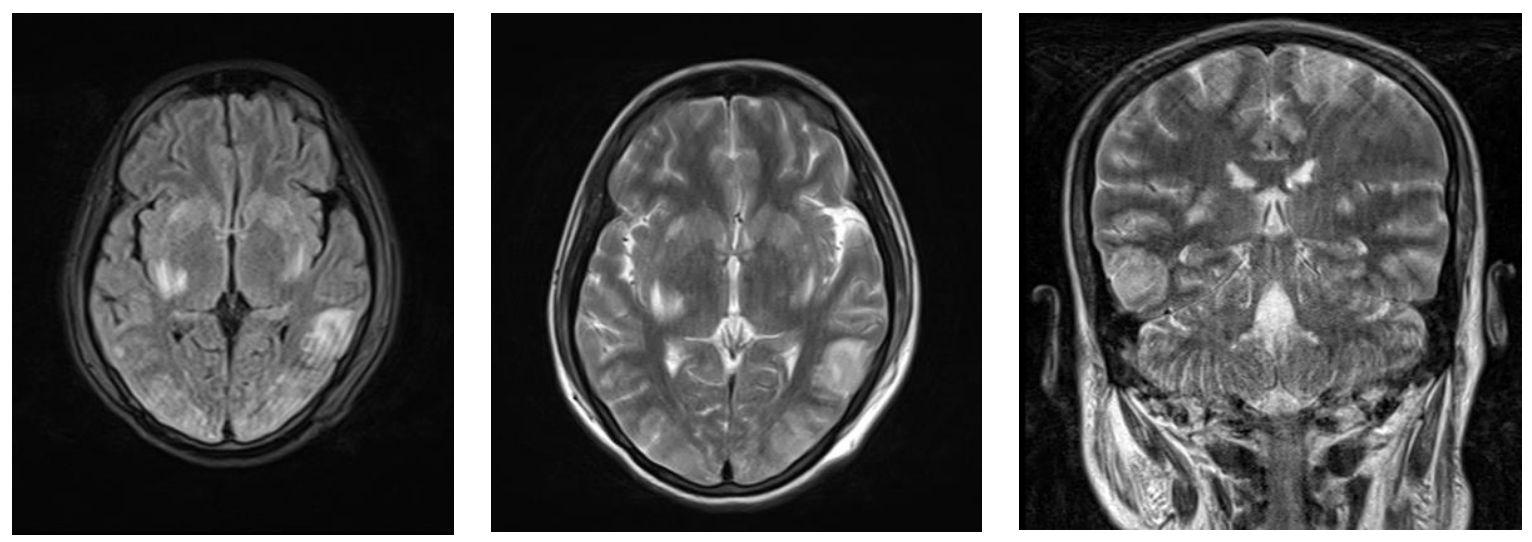

Fig. 1: Vasogenic Edema
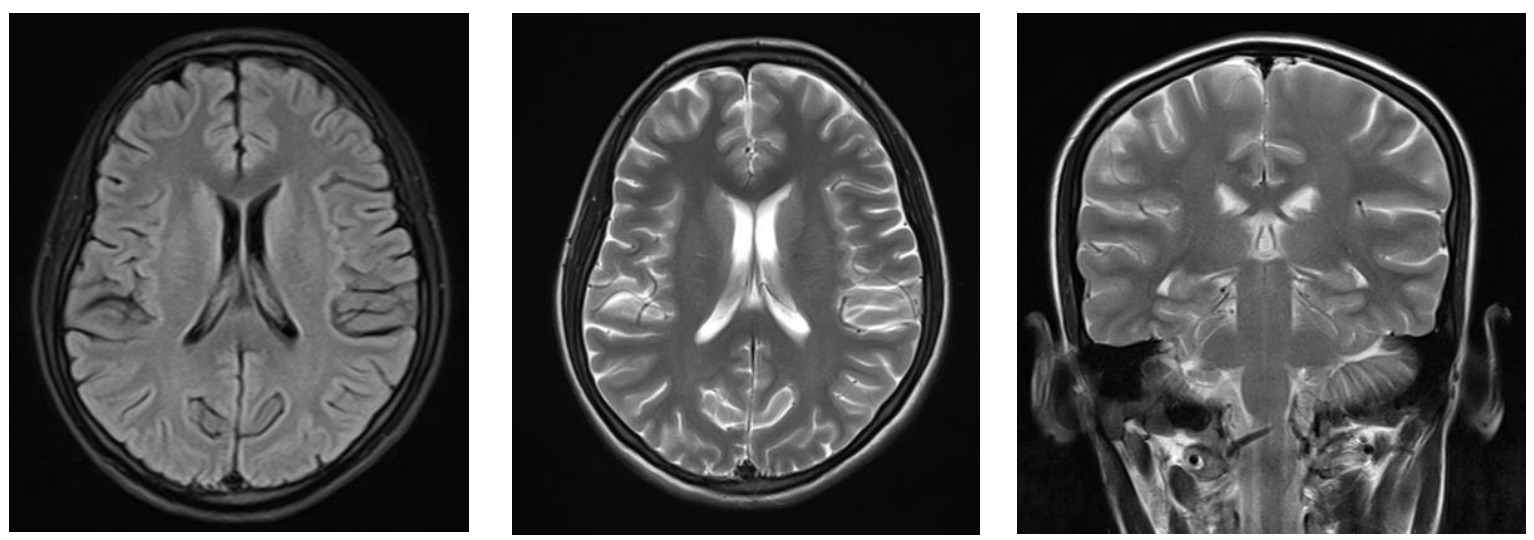

Fig. 2: Vasogenic Edema Relieved

\section{Discussion}

Preeclampsia if not treated promptly leads to development of seizures, a condition known as eclampsia. Moreover patients suffering from sickle cell anemia have higher chances of developing hypertension because of sickle cell nephropathy and hence women suffering from sickle cell disease have a higher chances of developing preeclampsia and hence eclampsia.

A pregnant woman during her period of gestation or immediately postpartum if has a seizure episode the 1 st differential diagnosis that comes to the mind of clinician is Eclampsia. PRES being rare is often missed causing delay in treatment leading to permanent damage in specific areas of brain in otherwise typically reversible condition.

Case series suggest that RPLS is more common in women, even when patients with eclampsia are excluded. . $^{1,4}$

Hypertensive disorders, renal disease and immunosuppressive therapies are risk factors for this disease. Pathogenesis of PRES is unclear, but appears to be due to disordered cerebral autoregulation and endothelial dysfunction. ${ }^{1}$ Normal autoregulation maintains constant cerebral blood flow over a range of systemic blood pressure by means of arterial constriction and dilatation. Once the upper limit of cerebral autoregulation is exceeded, arterioles dilate and cerebral blood flow increases in a pressure passive manner with rise in systemic blood pressure leading to hypo perfusion causing extravasation of fluid and blood products into brain parenchyma. Rate of blood pressure elevation is more important than the value of blood pressure itself. Alternatively it has been postulated that disordered autoregulation may lead to reactive vasoconstriction thereby resulting in local hypo perfusion, cytotoxic edema and cerebral infarction., ${ }^{4,3,7}$ It is also possible that cerebral infarctions which uncommonly occurs in RPLS could result from compression of micro circulation from the mass effect of vasogenic edema.

Endothelial dysfunction on the other hand leads to capillary leakage, blood brain barrier disruption and 
axonal swelling which then triggers vasogenic edema. ${ }^{[1,8]}$ Markers of endothelial cell dysfunction (LDH, Fibronectin, TPA, Thrombomodulin, endothelin 1 and vWF) rise prior to clinical syndrome and their levels correlate better with the extent of cerebral edema than does blood pressure changes. ${ }^{9-14}$

Unregulated vascular injury to blood brain barrier endothelium leads to edema, protein extravasation and fibrinoid necrosis which is evident radiographically. ${ }^{15}$

All patients suffering from PRES have characteristic features of headache, altered levels of consciousness, visual changes and seizures. ${ }^{[16]}$

Hypertension is a feature in the majority of RPLS patients, regardless of etiology $1,8,21,22,24,25$ With blood pressure lowering, patients will often improve dramatically. Except in cases of malignant hypertension, patients with RPLS often present with only moderate levels of hypertension; in most of cases, however, this still represents a significant increase above baseline levels.

The initial aim of treatment in malignant hypertension is to quickly bring down the diastolic blood pressure to around $100 \mathrm{mmHg}$ which should be achieved be achieved within 2 to 6hours. The maximum initial fall in blood pressure should not exceed 25 percent of the original value. ${ }^{26,27}$ More aggressive blood pressure lowering is generally unnecessary and may reduce the blood pressure below the autoregulatory range, possibly leading to ischemic events such as stroke or coronary disease. ${ }^{27}$

For patients with lower levels of hypertension, lowering blood pressure is also recommended to treat RPLS, but no specific guidelines are suggested as discussed for malignant hypertension above. Using clinical symptoms and any prior knowledge of baseline blood pressure as a guide, careful, incremental downward titration in 10 to 25 percent increments of the mean arterial blood pressure seems a reasonable approach.

The use of easily titratable parenteral agents such as nicardipine, labetalol, and nitroprusside are effective and safe in reducing the blood pressure to a desirable range. One caveat to the acute use of nitroprusside is the theoretical concern that it may paradoxically increase intracranial pressure through vasodilatation.

Except in patients suffering from eclampsia, majority of the patients with PRES and seizures are successfully treated with phenytoin. ${ }^{4,6,19}$ Other antiepileptics drugs can be used while considering the patient's coexisting medical disorders and prescribed drugs. ${ }^{30}$ While long-term follow-up studies are limited, seizure recurrence or epilepsy appears to be rare. In one case series of 127 patients who had recovered from an episode of RPLS, unprovoked seizures occurred in 8 patients over a median 3.2 years of follow up. ${ }^{31}$

Although some case series report continuation of therapy for one to three months, phenytoin can probably be safely tapered as symptoms and neuroimaging findings resolve, usually after one to two weeks. ${ }^{19}$ When recurrent, unprovoked seizures have occurred after recovery from an RPLS episode, it is reasonable to resume or initiate antiepileptic drug therapy. $29,31,32$

Management of patients with PRES with Eclampsia being the underlying cause is different from that due to other causes. Significant fall in blood pressure occurs once the baby and placenta are delivered. $\mathrm{MgsO}_{4}$ is superior to phenytoin and diazepam in managing seizures in such patients.

There are no specific diagnostic criteria for reversible posterior leukoencephalopathy syndrome (RPLS). In the appropriate clinical setting, clinicians should recognize the neurologic syndrome of headache, visual symptoms, confusion, and seizures and order a brain MRI. Diffusion-weighted imaging (DWI) plays an important role in diagnosis and prognosis.

Because the neuro radiographic findings after seizures or other neurologic conditions can be like those seen in RPLS, repeat neuroimaging may be necessary. With treatment, resolution of findings on neuroimaging within days to weeks is expected.

Other neurologic conditions like stroke, venous thrombosis, toxic or metabolic encephalopathy, demyelinating disorders, vasculitis, and encephalitis are to be considered while considering differential diagnosis for PRES. ${ }^{17,20,23}$

It is important to distinguish between RPLS and ischemic stroke, as the management protocols for hypertension are different.

In majority of the cases, almost all the features of PRES are reversed once the underlying cause is eliminated and blood pressure is controlled..$^{[1,18]}$ Radiological changes which are mostly reversible may not revert to normal for weeks even after the patient has recovered clinically.

Death although rare can occur due to progressive cerebral edema, intracerebral hemorrhage, or as a complication of the underlying condition., ${ }^{2,46}$

MRI findings of hyperintense signals on DWI and restricted diffusion on ADC mapping suggest cytotoxic as opposed to vasogenic edema; these findings seem to be predictive of irreversible infarction and in turn a worse prognosis. ${ }^{2}$ Extensive brain involvement, particularly in the brainstem depicts a poor prognosis.

\section{References}

1. Hinchey J, Chaves C, Appignani B, et al. A reversible posterior leukoencephalopathy syndrome. N Engl J Med 1996;334:494

2. Covarrubias DJ, Luetmer PH, Campeau NG. Posterior reversible encephalopathy syndrome: prognostic utility of quantitative diffusion-weighted MR images. AJNR Am J Neuroradiol 2002;23:1038.

3. Antunes NL, Small TN, George D, et al. Posterior leukoencephalopathy syndrome may not be reversible. Pediatr Neurol 1999;20:241. 
4. Ay H, Buonanno FS, Schaefer PW, et al. Posterior leukoencephalopathy without severe hypertension: utility of diffusion-weighted MRI. Neurology 1998;51:1369.

5. Provenzale JM, Petrella JR, Cruz LC Jr, et al. Quantitative assessment of diffusion abnormalities in posterior reversible encephalopathy syndrome. AJNR Am J Neuroradiol 2001;22:1455.

6. Stott VL, Hurrell MA, Anderson TJ. Reversible posterior leukoencephalopathy syndrome: a misnomer reviewed. Intern Med J 2005;35:83.

7. Mukherjee P, McKinstry RC. Reversible posterior leukoencephalopathy syndrome: evaluation with diffusion-tensor MR imaging. Radiology 2001;219:756.

8. Ito Y, Arahata Y, Goto Y, et al. Cisplatin neurotoxicity presenting as reversible posterior leukoencephalopathy syndrome. AJNR Am J Neuroradiol 1998;19:415.

9. Schwartz RB, Feske SK, Polak JF, et al. Preeclampsiaeclampsia: clinical and neuroradiographic correlates and insights into the pathogenesis of hypertensive encephalopathy. Radiology 2000;217:371.

10. Savvidou MD, Hingorani AD, Tsikas D, et al. Endothelial dysfunction and raised plasma concentrations of asymmetric dimethylarginine in pregnant women who subsequently develop pre-eclampsia. Lancet 2003;361:1511.

11. Easton JD. Severe preeclampsia/eclampsia: hypertensive encephalopathy of pregnancy? Cerebrovasc Dis 1998;8:53.

12. Lindenbaum J. An approach to the anemias. In: Cecil's Textbook of Medicine, Bennett JC, Plum F (Eds), Philadelphia 1996. P.823.

13. Rodgers GM, Taylor RN, Roberts JM. Preeclampsia is associated with a serum factor cytotoxic to human endothelial cells. Am J Obstet Gynecol 1988;159:908.

14. Blann AD, Taberner DA. A reliable marker of endothelial cell dysfunction: does it exist? Br J Haematol 1995;90:244.

15. Brightman MW, Klatzo I, Olsson Y, Reese TS. The blood-brain barrier to proteins under normal and pathological conditions. J Neurol Sci 1970;10:215.

16. Fugate JE, Claassen DO, Cloft HJ, et al. Posterior reversible encephalopathy syndrome: associated clinical and radiologic findings. Mayo Clin Proc 2010;85:427.

17. Raps EC, Galetta SL, Broderick M, Atlas SW. Delayed peripartum vasculopathy: cerebral eclampsia revisited. Ann Neurol 1993;33:222.

18. Junna MR, Rabinstein AA. Tacrolimus induced leukoencephalopathy presenting with status epilepticus and prolonged coma. J Neurol Neurosurg Psychiatry 2007;78:1410.

19. Bakshi R, Bates VE, Mechtler LL, et al. Occipital lobe seizures as the major clinical manifestation of reversible posterior leukoencephalopathy syndrome: magnetic resonance imaging findings. Epilepsia 1998;39:295.

20. Oppenheim C, Galanaud D, Samson Y, et al. Can diffusion weighted magnetic resonance imaging help differentiate stroke from stroke-like events in MELAS? J Neurol Neurosurg Psychiatry 2000;69:248.

21. Henderson RD, Rajah T, Nicol AJ, Read SJ. Posterior leukoencephalopathy following intrathecal chemotherapy with MRA-documented vasospasm. Neurology 2003;60:326.

22. Ito $\mathrm{Y}$, Niwa $\mathrm{H}$, Iida $\mathrm{T}$, et al. Post-transfusion reversible posterior leukoencephalopathy syndrome with cerebral vasoconstriction. Neurology 1997;49:1174.

23. Casey SO, Sampaio RC, Michel E, Truwit CL. Posterior reversible encephalopathy syndrome: utility of fluidattenuated inversion recovery MR imaging in the detection of cortical and subcortical lesions. AJNR Am J Neuroradiol 2000;21:1199.

24. Kupferschmidt H, Bont A, Schnorf H, et al. Transient cortical blindness and bioccipital brain lesions in two patients with acute intermittent porphyria. Ann Intern Med 1995;123:598.

25. Kaplan, NM. Hypertensive crises. In: Clinical Hypertension, 7th, Kaplan NM (Ed), Williams \& Wilkins, Baltimore 1998. P.265.

26. Vaughan CJ, Delanty N. Hypertensive emergencies. Lancet 2000;356:411.

27. Ledingham JG, Rajagopalan B. Cerebral complications in the treatment of accelerated hypertension. Q J Med 1979;48:25.

28. Neutel JM, Smith DH, Wallin D, et al. A comparison of intravenous nicardipine and sodium nitroprusside in the immediate treatment of severe hypertension. Am J Hypertens 1994;7:623.

29. Arnoldus EP, Van Laar T. A reversible posterior leukoencephalopathy syndrome. N Engl J Med 1996;334:1745; author reply 1746 .

30. Eberly AL, Anderson GD, Bubalo JS, McCune JS. Optimal prevention of seizures induced by high-dose busulfan. Pharmacotherapy 2008;28:1502.

31. Datar S, Singh T, Rabinstein AA, et al. Long-term risk of seizures and epilepsy in patients with posterior reversible encephalopathy syndrome. Epilepsia 2015;56:564. 